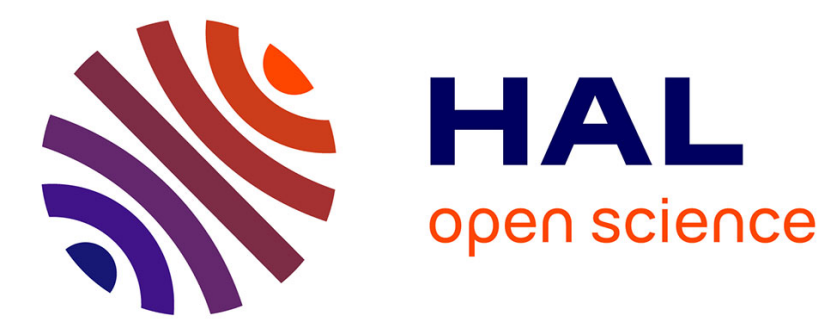

\title{
Dynamics of round jet impingement
}

\author{
Vincent Jaunet, Matteo Mancinelli, Peter Jordan, Aaron Towne, Daniel \\ Edgington-Mitchell, Guillaume Lehnasch, Steve Girard
}

\section{To cite this version:}

Vincent Jaunet, Matteo Mancinelli, Peter Jordan, Aaron Towne, Daniel Edgington-Mitchell, et al.. Dynamics of round jet impingement. 25th AIAA/CEAS Aeroacoustics Conference, May 2019, Delft, Netherlands. 10.2514/6.2019-2769 . hal-02378573

\section{HAL Id: hal-02378573 https://hal.science/hal-02378573}

Submitted on 25 Nov 2019

HAL is a multi-disciplinary open access archive for the deposit and dissemination of scientific research documents, whether they are published or not. The documents may come from teaching and research institutions in France or abroad, or from public or private research centers.
L'archive ouverte pluridisciplinaire HAL, est destinée au dépôt et à la diffusion de documents scientifiques de niveau recherche, publiés ou non, émanant des établissements d'enseignement et de recherche français ou étrangers, des laboratoires publics ou privés. 


\title{
Dynamics of round jet impingement
}

\author{
V. Jaunet ${ }^{1}$ \\ M. Mancinelli ${ }^{1,4}$ \\ P. Jordan ${ }^{1}$ \\ A. Towne ${ }^{2}$ \\ D. Edgington-Mitchell ${ }^{3}$ \\ G. Lehnasch ${ }^{1}$ \\ S. Girard ${ }^{1}$ \\ ${ }^{1}$ Institut PPRIME, UPR-CNRS-3346, Université de Poitiers, ENSMA \\ ${ }^{2}$ Dept. of Mechanical Engineering, University of Michigan, Ann Arbor, MI 48109, USA \\ ${ }^{3}$ Dept. of Mechanical and Aerospace Engineering, Monash University, VIC 3800, Australia \\ ${ }^{4}$ CNES, Direction des Lanceurs, FRANCE
}

\begin{abstract}
The impingement of a round jet issued from a convergent nozzle is known to produce intense tonal noise. These tones are generated by a feedback process involving a KelvinHelmholtz instability wave and an upstream propagating wave. The frequency structure of these tones is experimentally explored by varying the jet Mach number from 0.7 to 1.5 and we observe evidence that neutral-upstream modes of the jet are active in the feedback process. Tone frequency predictions obtained using the intrinsic jet modes are compared to the standard model involving free-stream acoustic waves: the neutral waves model provides a convincing agreement with the experimental data.
\end{abstract}

\section{Introduction}

When a high subsonic or supersonic jet is directed normal onto a wall, it is well known that intense tonal noise is emitted [7, 12, 14, 22, The characteristics of the sound produced depend strongly on the jet Mach number, the plate size and the nozzle-plate spacing. An intensive and recent review of resonance in supersonic jets can be found in [2]

Ho and Nosseir [8] found evidence that, for subsonic jets, these tones are produced by a feedback process involving a hydrodynamic perturbation convected downstream onto the plate and an acoustic wave propagating upstream towards the nozzle to close the loop. Similar observations were made later by Powell [15] for the impingement of an underexpanded jet, who hence proposed a simple model to predict the impingement tone frequencies, similar to his screech prediction model [13].

On the other hand, Tam and Ahuja [20] proposed an alternative feedback mechanism. They suggested that the feedback involves upstream-propagating waves, that are intrinsic acoustic modes of the jet, instead of the free-stream acoustic waves. These modes had been described earlier by Tam and Hu [19], and their characteristics can be obtained by modeling the jet as a vortex-sheet. Tam and Ahuja [20] obtained a good agreement between experimental and theoretical predictions for subsonic Mach numbers. These results showed that the flow can support different feedback processes to resonate. Recently, Towne et al. [21] and Schmidt et al. 17] showed that these intrinsic jet modes are responsible for the weak resonance observed in the high subsonic jet potential core and Jordan et al. 9] revealed their involvement in the generation of intense tonal noise of grazing subsonic jets. Recently, Edgington-Mitchell et al. [3] confirmed experimentally, in a recent publication, the presence of such waves in screeching circular jets, and Mancinelli et al. [11] successfully predicted the screech mode frequencies of the axisymmetric screech tones using the intrinsic modes dispersion relation. These results confirmed Shen and Tam [18] conjecture that these upstream-modes play a role in the feedback process of screech.

The role of these waves in the case of impinging round and planar ideally expanded supersonic jets was recently highlighted by Bogey and Gojon [1] and Gojon et al. [4 respectively using numerical simulations. The present paper aims at pursuing the effort in studying the possible involvement of the intrinsic jet modes

Copyright (c) 2019 by The Authors. Published by the American Institute of Aeronautics and Astronautics, Inc. with permission.

1 of 11 
in the feedback process. We therefore performed an experimental campaign to obtain detailed parametric data in function of both Mach number and jet-plate position, in order to offer a more general view of the phenomenon. Moreover, a quantitative prediction method based on satisfying resonance conditions, following Jordan et al. 9], is proposed to compute possible resonant frequencies and compare the results with the experimental observations. The agreement obtained underpins the fact that these waves are active in the feedback process.

The paper is organized as follows. Section II is dedicated to the experimental set-up presentation, followed by a description of the results obtained in the section [II]. Then, section [IV] present the methodology that has been used to compute the resonant frequencies. A discussion is provided in section VI.

\section{Experimental set-up}

The experiments were conducted in the SUCRE (SUpersoniC REsonance) anechoic wind tunnel, located at the PROMETEE platform, PPRIME Institute, Poitiers. The experimental set-up consists of an axisymmetric jet, issuing from a $D=0.01 \mathrm{~m}$ convergent-straight nozzle, and impinging on a flat plate of large dimensions compared to the nozzle diameter. We used relatively thin nozzle lips of thickness equal to $0.03 D$. The distance from the nozzle to the plate is varied from $H=2 D$ to $H=8 D$ using an automated displacement system. A sketch of the set-up, presenting the coordinate system used in the paper, is given in figure 1 .

The nozzle is fed by desiccated and compressed air at 200bar, expanded to the desired stagnation pressure. The stagnation temperature of the flow is maintained equal to the room temperature $\left(T_{0}=293 \mathrm{~K}\right)$ via an electrical heating element and a PID controller. This temperature was chosen to avoid condensation and freezing on the nozzle even at the highest possible Mach number. The nozzle pressure ratio (NPR), i.e. ratio of stagnation to ambient pressure $P_{0} / P_{a}$ is monitored via a computer controller to offer stabilized fully expanded jet Mach numbers in the $M_{j}=[0.7-1.5]$ range. The fully expanded jet Mach number $M_{j}$ is defined via the classical isentropic relations:

$$
\frac{P_{a}}{P_{0}}=\left(1+\frac{\gamma-1}{2} M_{j}^{2}\right)^{-\frac{\gamma}{\gamma-1}},
$$

where $\gamma$ is the specific heat ratio. The associated Reynolds number, based on the fully expanded jet quantities, $R e=\rho_{j} U_{j} D / \mu$ varied in the $[1.37-5.5] \times 10^{5}$ range, which combined the relatively low contraction ratio of the nozzle (the stagnation chamber to nozzle diameter ratio was chosen around 6 for space saving considerations), ensures the turbulent state of the jet mixing layers.

A microphone is used to record the near-field acoustic signature of flow. It is located in the nozzle exit plane at a radial position $r / D=1$. The pressure sampling frequency was set at $200 \mathrm{kHz}$, above the cut-off frequency of the microphone to satisfy Shanon's criteria. This provides a maximum resolved Strouhal number $S t=f D / U_{j}$, based on the nozzle diameter $D$ and the fully expanded jet velocity $U_{j}$, in the $[2.34-8]$ range, well above the frequencies of interest in this paper. The fully expanded jet velocity $U_{j}$ is defined by :

$$
U_{j}=\sqrt{\gamma r T_{0}} \frac{M_{j}}{\sqrt{\left(1+\frac{\gamma-1}{2} M_{j}^{2}\right)}},
$$

where $T_{0}$ is the stagnation temperature of the flow. A total number of $6 \times 10^{6}$ samples were acquired at each $\left(M_{j}, H\right)$ couple investigated, providing 30 seconds of signal which is orders of magnitude larger than the longest convective time of interest here. This ensures the convergence of the statistical quantities presented in the paper.

\section{Jet/plate interaction modes}

In figure 2, we present maps of the power spectral density (PSD) of acoustic pressure as a function of Strouhal number $S t=f D / U_{j}$ and fully expanded jet Mach number $M_{j}$ for various nozzle-plate distances. These specific distances were selected because they highlight the key features that can be observed in all cases.

For low distances (see figure 2a), the dominant peaks possess relatively high Strouhal number $(S t>0.5)$ and their frequency decreases with Mach number. As expected, mode staging is visible around $M_{j}=1.05$, 


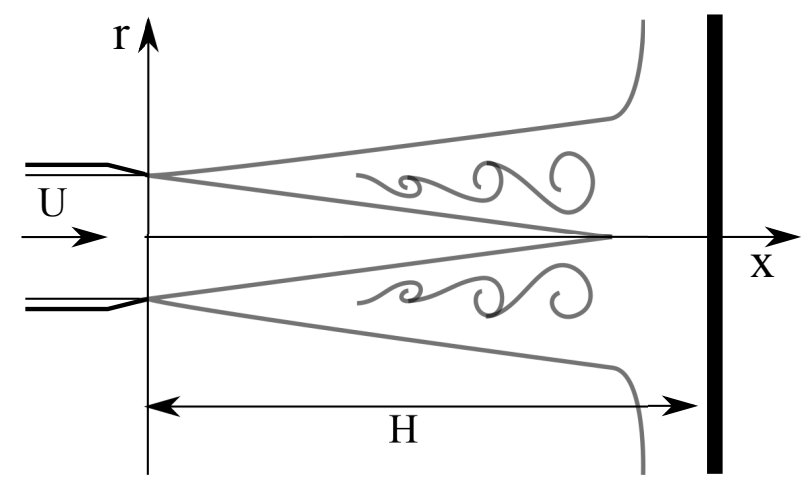

Figure 1: Sketch of the experimental arrangement.

where the dominant mode suddenly switches to lower Strouhal number with increasing Mach number. As observed in the subsonic regime [9], the PSD maps show bands of overall lower energetic level at certain Mach numbers, for example at $M_{j}=1.28$ and at $M_{j}=1.35$ in figure 2a. These regions may be linked to the zones of silence described by Henderson et al. [6]. Many other isolated peaks arise from the background noise level, but they do not show a clear organization or pattern.

For $H / D=4$ (figure 2b), a clearer organization of the dominant peaks can be seen. First of all, some peaks can be tracked over a wider range of Mach numbers than for $H / D=2$ and a hierarchical organization of different modes, in terms of Strouhal number, starts to emerge. This organization is increasingly clearer as the jet-plate distance is further increased. If one follows a single specific peak, around $S t=0.3$ and $M_{j}=1.0$, in figures 2c or 2d there is a given Mach number beyond which the peak extinguishes. The extinction locations of all the peaks in the $\left(M_{j}, S t\right)$ plane forms a curve and several of these curves can be identified in the maps of figures $2 \mathrm{c}$ or $2 \mathrm{~d}$. The similarity of this behavior to what was observed by Jordan et al. 9] indicates that the neutral acoustic modes of the jet might play a non-negligible role in the generation of underexpanded jet impingement tones.

In addition to the impingement tone, screech tones [13, 16, are clearly visible in figure 2d, where the signature of the A1 and A2 modes can be seen for $1.0<M j<1.15$ and the B mode arises for $M_{j}>1.15$. Screech modes only appears when the plate is far enough from the nozzle, as already observed by Henderson and Powell [5]. If the plate is too close to the nozzle, it prevents the formation of the shock cell pattern which is a necessary ingredient of the screech generation.

\section{Resonance models}

In this section we present the models that are being investigated in order to predict the resonance frequency of the impingement tones. All models involve two waves: the first one is a downstream travelling wave, which will be denoted by a superscript $(\cdot)^{+}$, and the second one is an upstream travelling wave, which will be denoted by a superscript $(\cdot)^{-}$. We restrict ourselves to only consider the real part of the wavenumbers of those waves. This means that the Kelvin-Helmholtz instability is modeled here as a neutral wave, but it is expected that this simplification should not greatly affect the resonance frequency predictions [9].

\section{A. Resonance criterion}

For resonance to occur over a distance $L$, the two wavenumbers of the two waves must satisfy the following relations:

$$
\Delta k_{r}=k_{r}^{+}-k_{r}^{-}=\frac{2 n \pi+\phi}{L},
$$

where the underscript $(\cdot)_{r}$ denotes the real part of the wavenumber, $n$ is an integer and $\phi$ is a phase lag which can be obtained from the reflection coefficients of the waves at the boundaries [9]. Although these reflection coefficients are unknown, we use the two extreme values of $\phi=0$ and $\phi=\pi$ in the rest of the paper. This means that we consider two types of end conditions for the resonance: either we consider both the plate and the nozzle to act as rigid walls $(\phi=0)$ or we assume one rigid and one soft walls $(\phi=\pi)$. 


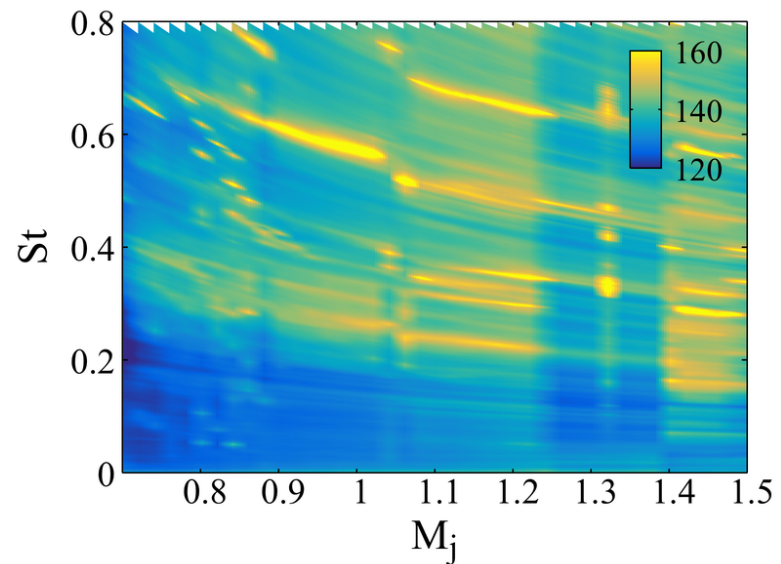

(a) $H / D=2$

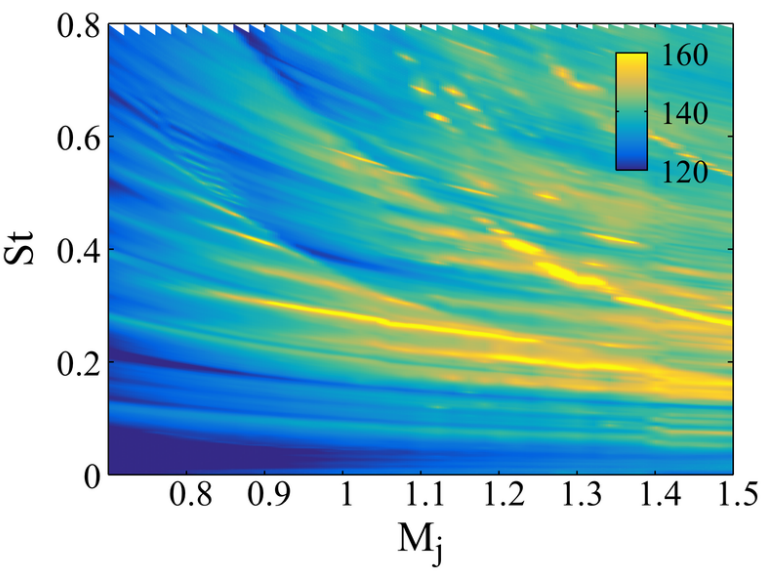

(c) $H / D=6$

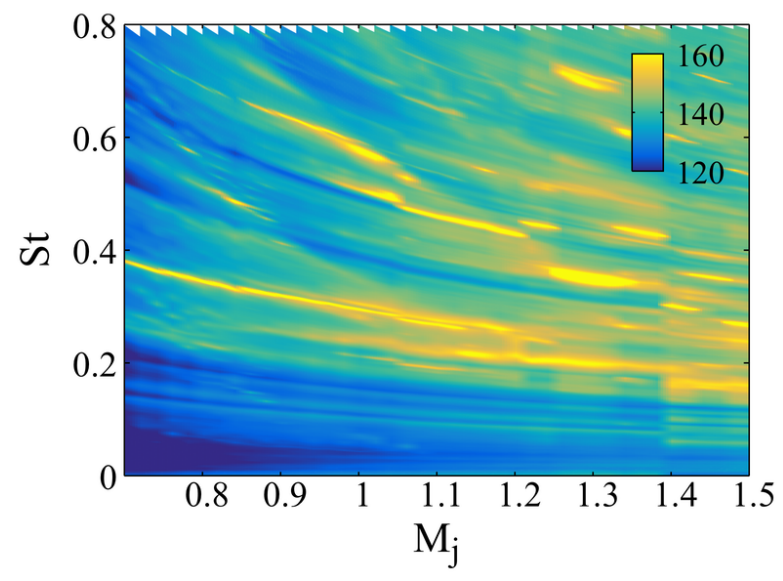

(b) $H / D=4$

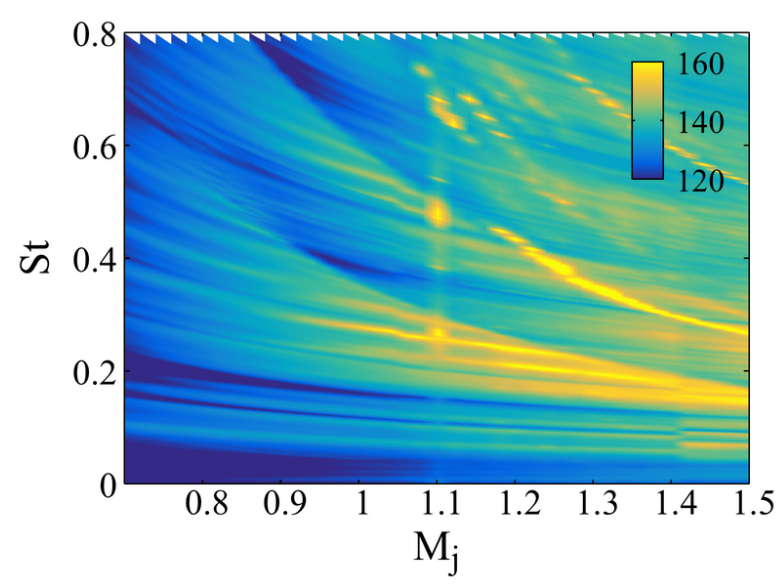

(d) $H / D=8$

Figure 2: PSD of the acoustic data as a function of the jet Mach number for various plate positions. 


\section{B. Modeling the Kelvin-Helmoltz wave}

Several models are used for the Kelvin-Helmoltz $(\mathrm{K}-\mathrm{H})$ wave. It is first assumed to be a non-dispersive wave with a phase velocity of $0.5 M_{j}$ or $0.8 M_{j}$. These convection velocities correspond to values experimentally observed [10, 23], and to values close to the one computed using a vortex-sheet model, respectively. The last model considered here for the $\mathrm{K}-\mathrm{H}$ wave uses the dispersion relation obtained from the vortex-sheet model of he jet.

The figure 4 presents the dispersion relations for all these models. The dispersive nature of the K-H wave computed using the vortex-sheet model is clear in this figure.

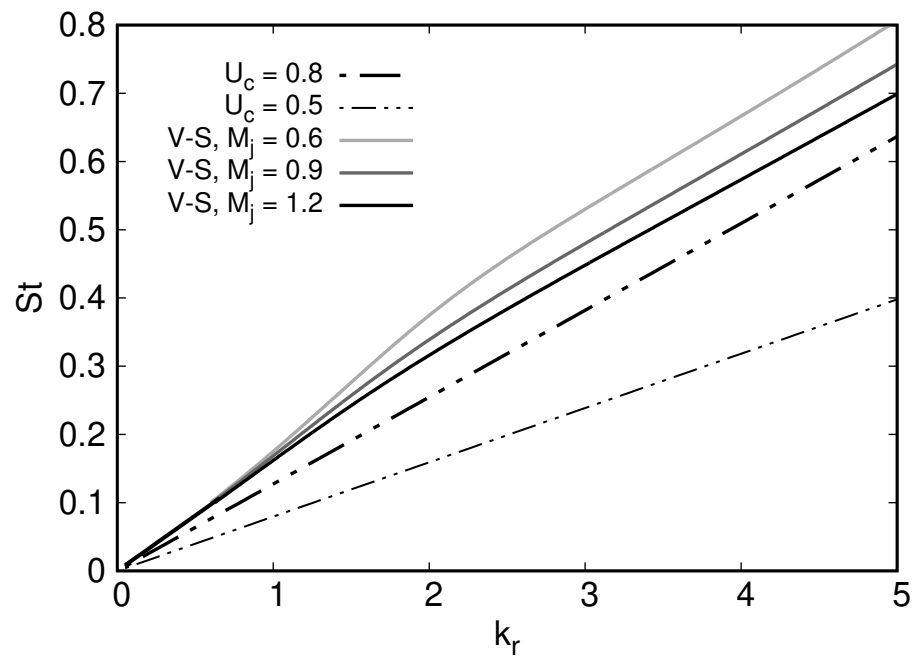

Figure 3: Dispersion relations of the K-H waves used in the study. Only the real part of the wavenumber is presented.

\section{Resonance between K-H and free-stream acoustic waves}

The first resonance model we consider in this paper was proposed by Powell [15] and involves sound waves as feedback mechanism. Powell [15] argued that the main impinging tone period is a fraction of sum of the travel time of convected disturbances in the jet mixing layer, i.e.the Kelvin-Helmholtz (K-H) instability wave, and the time for an acoustic wave, created by the impingement of the $\mathrm{K}-\mathrm{H}$ vortex onto the plate, to reach back the nozzle. This can be written in non-dimensionalized terms:

$$
\frac{N+p}{S t}=\frac{M_{j}}{D}\left(\frac{h_{c}}{M_{c}}+h_{a}\right),
$$

where $M_{c}=U_{c} / a$ is convective Mach number of the downstream disturbance, $h_{c}$ and $h_{a}$ represent the travelling distance of both the downstream travelling wave and the free-stream acoustic wave. $N$ corresponds to the number of observed disturbances between the nozzle lip and the plate at a given instant of the feedback loop and $p$ is a phase-lag parameter usually comprised between -0.5 and 0 [23]. Powell [15] introduced this term to account for the fact that hydrodynamic-acoustic interactions may not be perfectly in phase at both ends of the feedback loop. Assuming $h_{c} \simeq h_{a}=H$, the equation 4 can be rewritten in the following form:

$$
k_{c}^{+}-k_{a}^{-}=\frac{2 \pi(N+p)}{H},
$$

where $k_{a}^{-}$and $k_{c}^{+}$are the real part of the wavenumbers of the acoustic and the Kelvin-Helmholtz waves respectively. As can be seen, this relation is identical to the general wave resonance equation 3 described above. We see that the phase lag $p$ introduced by Powell [15] corresponds to the end conditions (i.e. the reflection coefficients) imposed at the boundaries. 
The method of determination of the frequency at which a resonance can occur is depicted in figure 4 where the dispersion relations of both the Kelvin-Helmholtz wave and the upstream-propagating free-stream acoustic wave are plotted for $M_{j}=0.6,0.9$ and 1.2. There is a possible resonance, at a given Mach number and given jet-plate distance at all the frequencies where the relation 3 is satisfied. The three first Strouhal numbers at which this happens for $M_{j}=1.2$, and a given arbitrary nozzle-plate distance $L$, are represented by the horizontal arrows in figure 4.

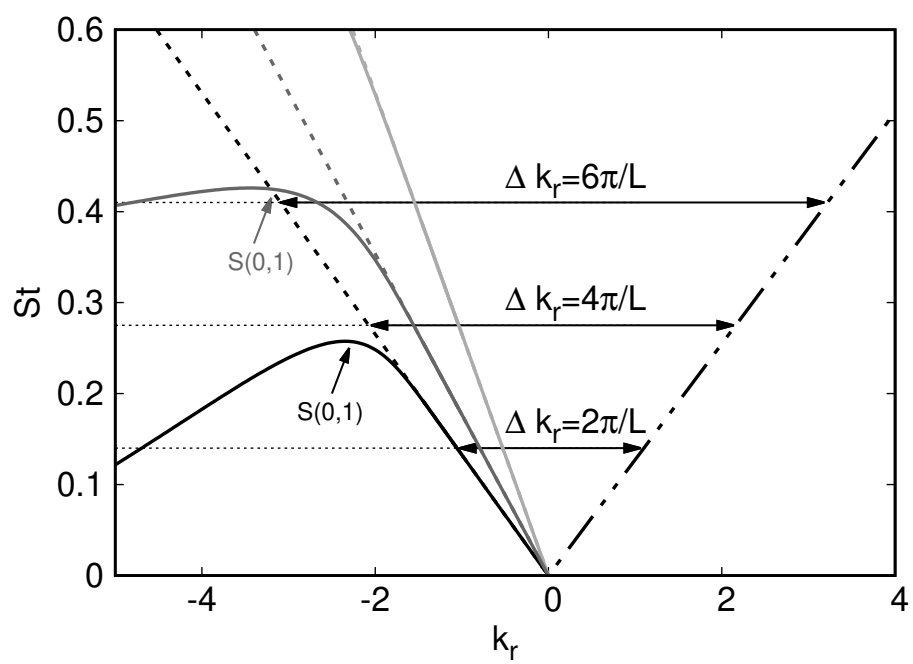

Figure 4: Dispersion relations used in the study: acoustic $k_{p}^{-}$jet modes $(-)$, upstream free-stream acoustic waves (-- ) and Kelvin-Helmholtz waves (-.-). Light gray lines corresponds to $M_{j}=0.6$, gray lines to $M_{j}=0.9$ and black lines to $M_{j}=1.2$.

\section{Resonance between $\mathrm{K}-\mathrm{H}$ and intrinsic jet modes}

The second resonance model we consider in this paper involves an acoustic jet mode, denoted $k_{p}^{-}$, in lieu of a free-stream acoustic wave to close the feedback loop, as proposed by Tam and Ahuja [20]. These waves were first described by Tam and Hu [19] using a vortex sheet model of the jet. They are neutrally stable, upstream-propagating and have spatial support both outside and inside of the jet. This make them perfect candidates to close the feedback loop. It is possible to hierarchically order these waves by their azimuthal wavenumber and their radial order. In the remainder of this paper, we only consider the lowest azimuthal modes (hence, axisymmetric modes) and lowest radial order (the spatial support of the waves only show one node). Typical dispersion relations of the axisymmetric jet modes for $M_{j}=0.6,0.9$ and 1.2 are also shown in figure 4. For the highest Mach number (black lines), and this is true for all supersonic Mach Numbers, the dispersion relation shows a maximum Strouhal number above which no neutral waves are supported by the jet. This means that, at supersonic Mach number, no resonance involving neutral waves should occur above this frequency. The limit is indicated by the point $S(0,1)$ in figure 4 Note, that on the left hand side of this point, the neutral waves have a positive group velocity and therefore cannot be involved in the feedback process of the resonance, we hence discard these waves in the following.

At $M_{j}=0.9$ (dark-gray lines), and similar behavior is observed for $0.82<M_{j}<1.0$, the dispersion relation also shows a maximum (gray $S(0,1)$ indication), but this is only a local maximum (see Towne et al. 21] for a more precise discussion). Upstream-propagative neutral modes can actually be found at higher Strouhal number. These high frequency neutral waves have been shown to behave more like duct modes, and have very limited spatial support outside of the jet which disqualify them as possible candidates for the feedback mechanism [9]. Again, for these Mach number range, we only retain the waves at the right-hand side of the $S(0,1)$ point.

For Mach numbers below $M_{j}=0.82$, there can only be found $k_{p}^{-}$waves for all Strouhal number and no maximum Strouhal number can be observed in the dispersion relation. These waves are dispersive: their dispersion relation deviates from a straight line. Again, for these Mach numbers, high frequency waves also 
behave as duct modes so that we discard them.

The determination of the resonance frequency follows the same procedure as for the first model. The only difference is the use of the $k_{p}^{-}$dispersion relation. As can be seen in figure 4 there is a wide range of frequencies where the dispersion relation of the acoustic jet mode is not very different from the one of the free-stream acoustic waves. Hence, we can expect both model to provide agreeing resonant frequency in this frequency range. This is what is depicted, for example, in figure 4 both model predict the same frequency for the first possible resonance. On the other hand, the two models would start to disagree, for example, when predicted resonance occur close to or beyond the location of the maximum frequency $S(0,1)$.

\section{Comparison with experimental data}

We provide in figure 5 the predictions obtained assuming the resonance occur between free-stream acoustic waves and the $\mathrm{K}-\mathrm{H}$ wave. We plot the predicted resonances using the first six values of $n$. As shown by the red lines, Powell [15] model based on resonance of free-stream acoustic modes is able to predict some of the dominant peaks for all cases but is never close to all of them at the same time. As can be seen by comparing the thin and thick red lines, adjusting the phase-lag parameter $\phi$ in relation 3 provides almost identical previsions with only shifted frequencies (the distance between the curves remain the same). Changing the $\mathrm{K}-\mathrm{H}$ wave phase speed does not change much to the overall representativity of the model. In a similar manner to the phase-lag parameter, the K-H phase speed simply shifts the predicted resonance towards higher frequencies for faster waves.

It must be noted that there will always be a combination of phase-lag and the K-H phase speed values that will match a given frequency in the domain. Hence, any peak in the spectrum can technically be predicted using this model. It is however impossible with this model to well predict all the dominant peaks at the same time using a given and fixed set of parameters.

Moreover, a drawback of this model is that it predict peaks where no resonance is observed, or when the peak has extinguished in the experimental data.

We must note, however, that at low Mach numbers $\left(M_{j}<0.8\right)$, this model provides predictions that follows particularly well the trend of peaks in the experimental data.

The predictions using the model involving the intrinsic jet modes are presented in figure 6. The upper Strouhal number limits above which the intrinsic jet modes are evanescent are overlayed as black lines on the maps of figure 6. At a given Mach number, the lowest line correspond to the limit of existence of mode $(m=0, n=1)$ (i.e. $S(0,1))$, followed by the limits of modes $(m=1, n=1),(m=0, n=2)$ and $(m=1, n=2)$ for increasing Strouhal number. As can be seen, there is almost a perfect match between the tone extinction and the location of $S(0,1)$. As for the screech tones [3, 11], this is a strong indication of the involvement of the intrinsic jet modes in the impingement resonance mechanism.

On the left hand side pictures of figure 6, the predictions are obtained using a modeled K-H wave of constant phase speed of $0.5 M_{j}$ (a value close to experimental observations). As for the previous resonance model (K-H and freestream acoustic), the predicted frequencies do not follow the trends observed in the experimental data. For example, in figure 6a some measured peaks actually cross several prediction lines. This shows that this model is not a good representation of the phenomenon. On the other hand, the results on the right side of figure 6 are computed using the $\mathrm{K}-\mathrm{H}$ dispersion relations provided by the vortex sheet model and, for all jet-plate distances presented here, it is clear that this model provides predictions with an improved accuracy. Although none of the predictions perfectly collapse onto a measured peak, this model is capable of following the experimental trends across a wide range of Mach numbers and jet/plate distances. Remembering that the predictions are obtained using a phase-only criterion, the actual reflection coefficient being unknown, this result is a clear indication that the impinging underexpanded jet tones may involve both the K-H wave and an intrinsic jet modes.

\section{Conclusions and Perspectives}

An experimental campaign has been performed to evaluate the role of the neutral upstream waves in the impingement tones of axisymmetric jet flows. Tone frequency predictions were calculated using a simple jet-plate model in which the jet is modeled as a vortex-sheet. Both the nozzle and the plate are seen, in this

7 of 11 

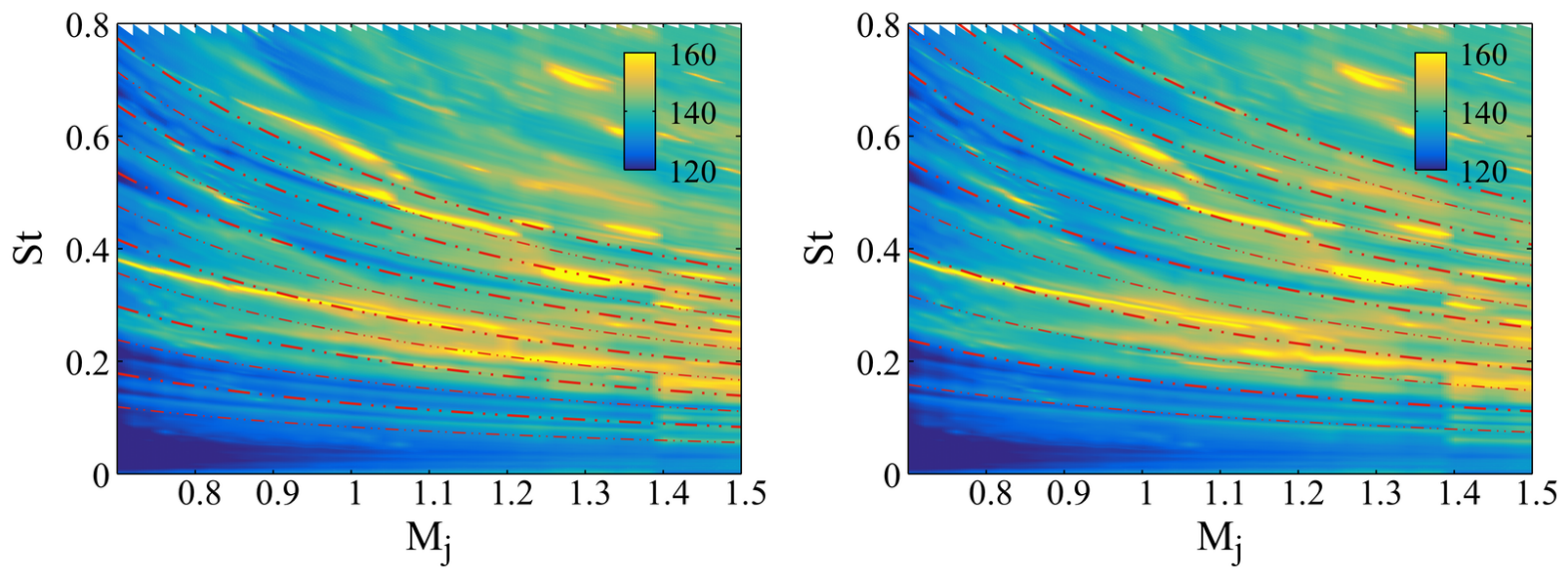

(a) $H / D=4$
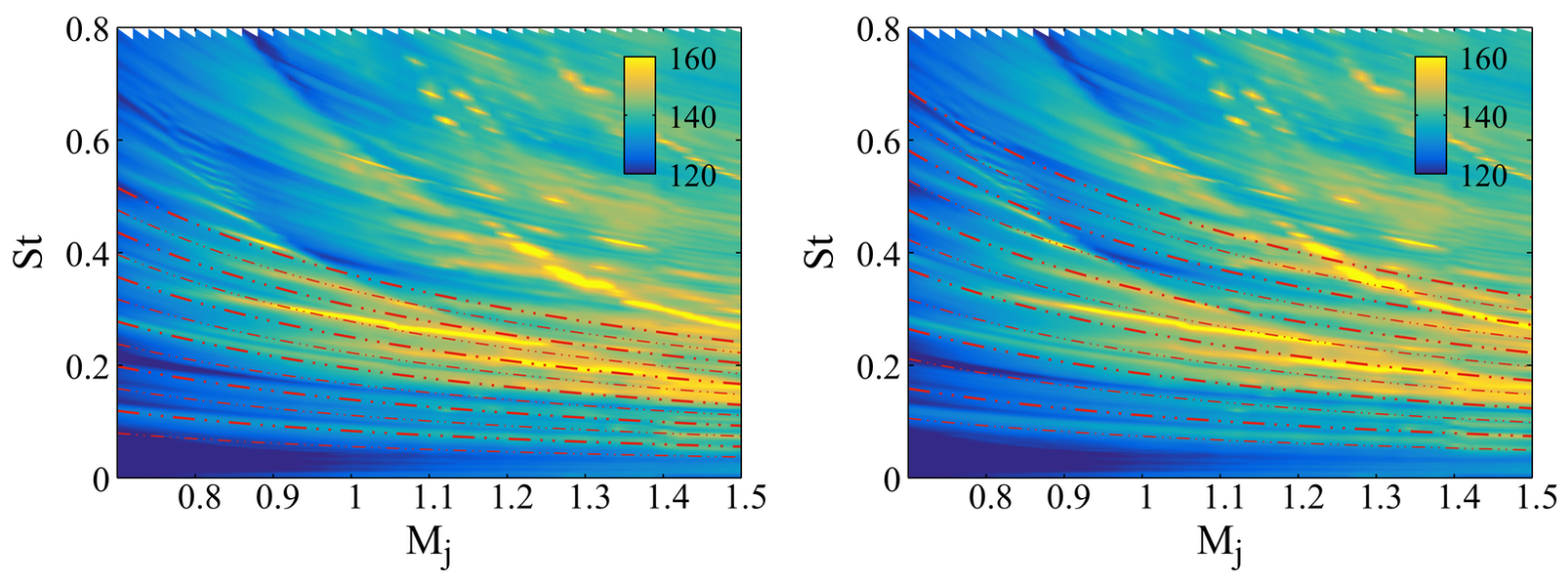

(b) $H / D=6$
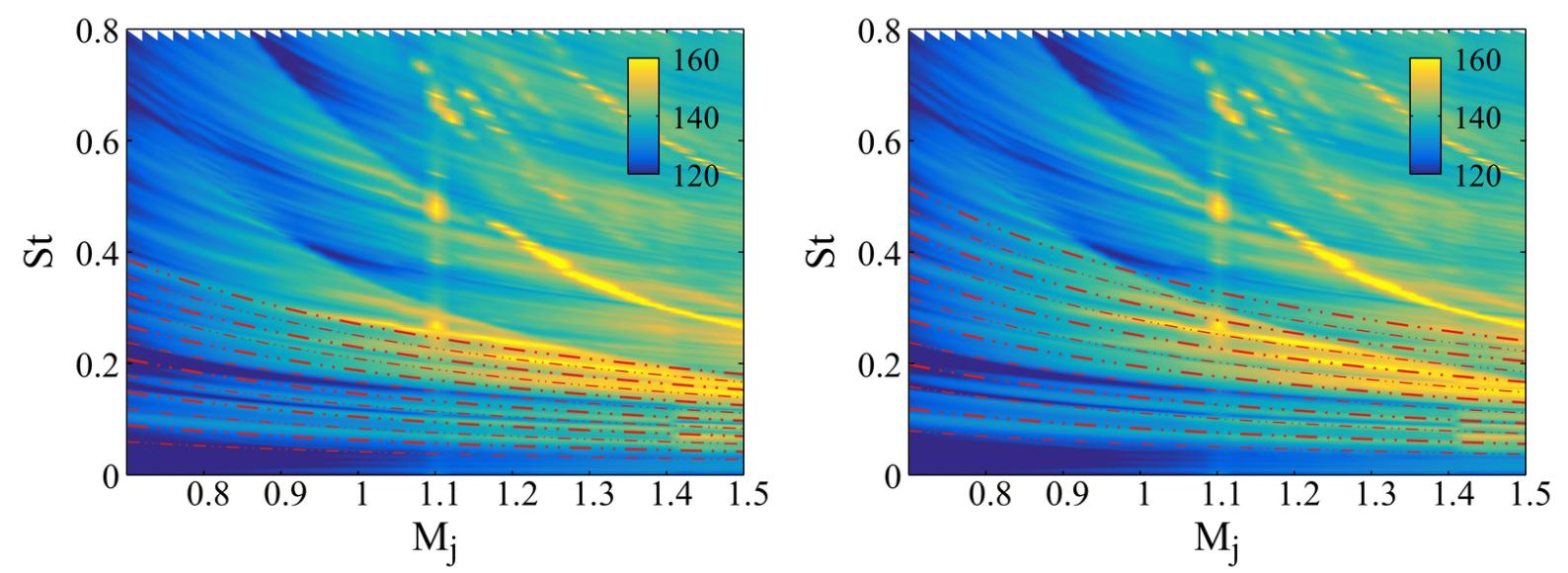

(c) $H / D=8$

Figure 5: Power spectral density maps of the acoustic data, in $d B / S t$ for various plate positions overlayed with the frequency predicted using free-stream acoustic waves. The $\mathrm{K}-\mathrm{H}$ waves is modeled using a constant phase speed of $0.5 M_{j}$ (left) and $0.8 M_{j}$ (right). Thick lines correspond to $\phi=0$ and thin lines to $\phi=\pi$. 

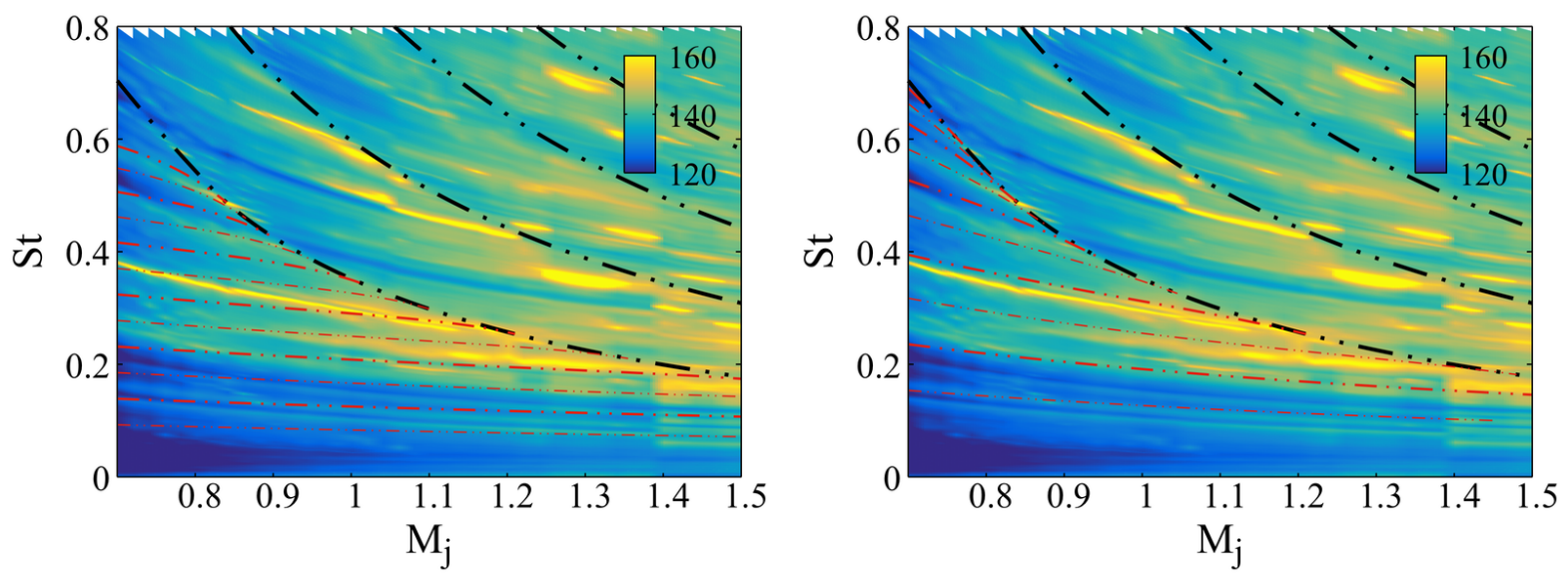

(a) $H / D=4$
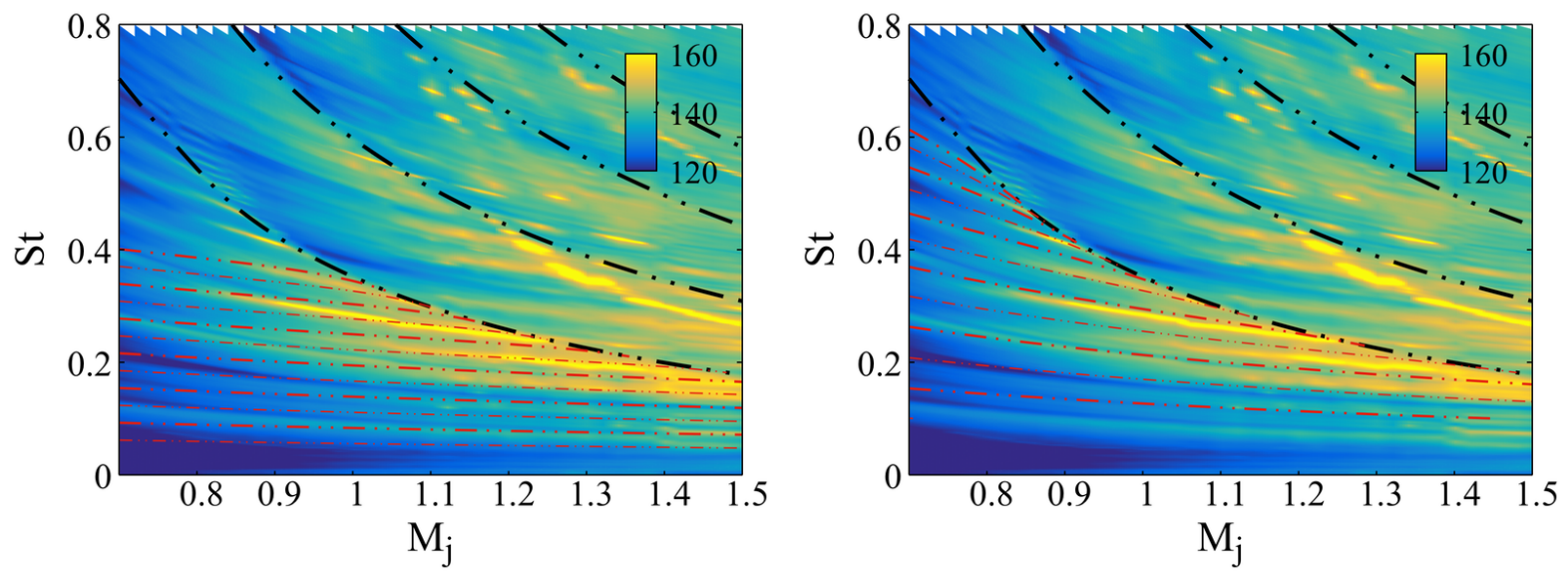

(b) $H / D=6$
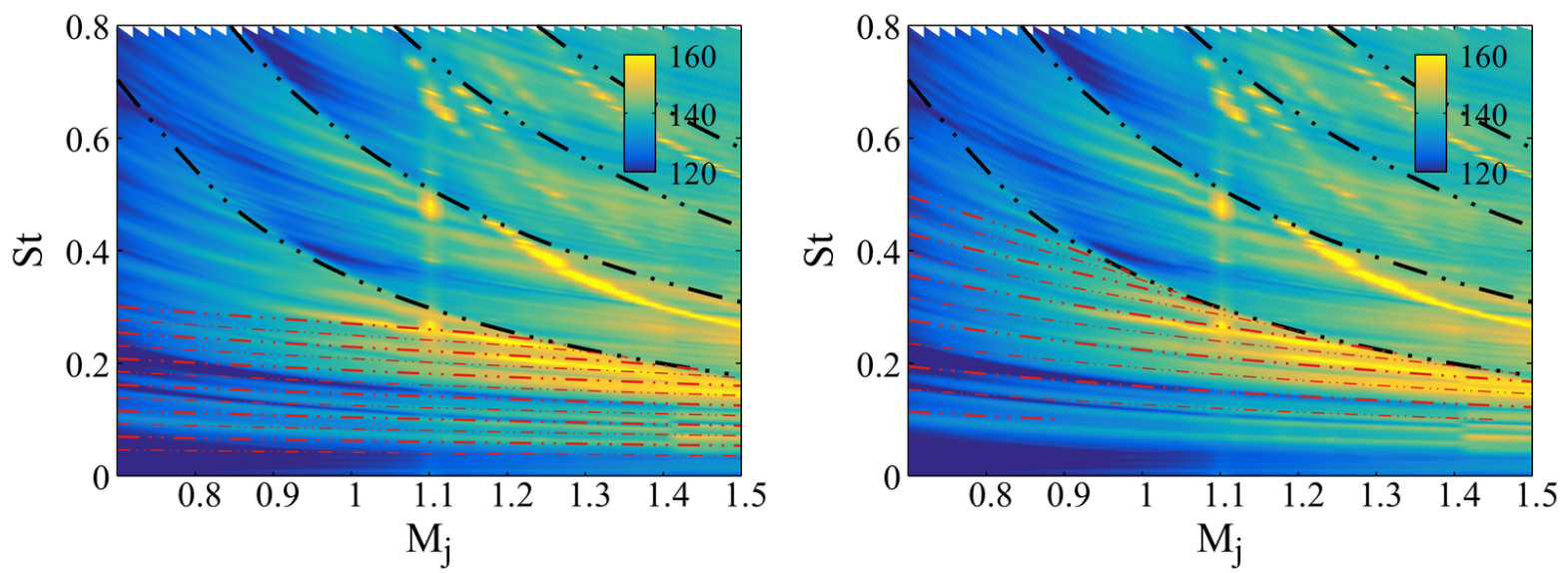

(c) $H / D=8$

Figure 6: Power spectral density maps of the acoustic data, in $d B / S t$ for various plate positions overlayed with the frequency predicted using intrinsic jet modes as upstream wave. The $\mathrm{K}-\mathrm{H}$ waves is modeled using a constant phase speed of $0.5 M_{j}$ (left) and the vortex-sheet model (right). Black lines correspond to the upper limit of exitence of the firt four intrinsic modes. Thick red lines correspond to $\phi=0$ and thin red lines to $\phi=\pi$. 
model, as end-conditions for the downstream K-H wave and the upstream neutral wave to exchange energy. Although, this is clearly an overly simplified view of the jet flow: none of the role played by the internal shock structure of the jet (see [6]), nor the design of the external geometry of the nozzle (see [24]) are taken into account ; some evidence were found in favor of this simplified model.

Firs of all, the agreement between the frequencies of the tones extinction on the one hand, and the frequencies beyond which no upstream waves should be observed on the other hand, provides a striking evidence that the neutral upstream waves are involved in the feedback process, as originally proposed by Tam and Ahuja 20]. This result confirms the observations of Bogey and Gojon [1 for an ideally expanded $M_{j}=1.5$ jet and shows that this model remains valid for underexpanded jet cases.

Secondly, a phase only criteria was used to compute the frequency at which those two waves may resonate, as in Jordan et al. 9] and Mancinelli et al. [11, and a relatively good agreement was obtained between predicted and observed resonance. An improvement of the predictions was observed when using the dispersion relation obtained via the vortex-sheet model for both the downstream and the upstream wave is clearly beneficial for the predictions. This is due to the dispersive nature of both the $\mathrm{K}-\mathrm{H}$ and the intrinsic waves that modifies the frequency at which the resonance criterion is satisfied. This is again a clear indication that this simple model well represent the dynamics occurring in the resonance.

If the waves at play in the impinging underexpanded jet tones seems now more clearly identified, and well modeled using a vortex-sheet dispersion relations, the prediction of their resonance still remains challenging. This is almost entirely due to the fact that the reflection coefficients, at the plate and at the nozzle, are unknown. This forces us to use a free parameter - the phase lag -, that technically allows to tune the model so that it matches any observations. A better knowledge of this reflection coefficient should provide valuable guidance in future modeling efforts of impingement tones.

\section{References}

${ }^{1}$ Christophe Bogey and Romain Gojon. Feedback loop and upwind-propagating waves in ideally expanded supersonic impinging round jets. Journal of Fluid Mechanics, 823:562-591, 2017.

2 Daniel Edgington-Mitchell. Aeroacoustic resonance and self-excitation in screeching and impinging supersonic jets-a review. International Journal of Aeroacoustics, page 1475472X19834521, 2019.

${ }^{3}$ Daniel Edgington-Mitchell, Vincent Jaunet, Peter Jordan, Aaron Towne, Julio Soria, and Damon Honnery. Upstream-travelling acoustic jet modes as a closure mechanism for screech. Journal of Fluid Mechanics, $855,2018$.

4 Romain Gojon, Christophe Bogey, and Olivier Marsden. Investigation of tone generation in ideally expanded supersonic planar impinging jets using large-eddy simulation. Journal of Fluid Mechanics, 808: 90-115, 2016.

5 B Henderson and A Powell. Experiments concerning tones produced by an axisymmetric choked jet impinging on flat plates. Journal of Sound and Vibration, 168(2):307-326, 1993.

${ }^{6}$ Brenda Henderson, James Bridges, and Mark Wernet. An experimental study of the oscillatory flow structure of tone-producing supersonic impinging jets. Journal of Fluid Mechanics, 542:115-137, 2005.

7 LF Henderson. Experiments on the impingement of a supersonic jet on a flat plate. Zeitschrift für angewandte Mathematik und Physik ZAMP, 17(5):553-569, 1966.

8 Chih-Ming Ho and Nagy S Nosseir. Dynamics of an impinging jet. part 1. the feedback phenomenon. Journal of Fluid Mechanics, 105:119-142, 1981.

9 Peter Jordan, Vincent Jaunet, Aaron Towne, André VG Cavalieri, Tim Colonius, Oliver Schmidt, and Anurag Agarwal. Jet-flap interaction tones. Journal of Fluid Mechanics, 853:333-358, 2018.

10 A Krothapalli, E Rajkuperan, F Alvi, and L Lourenco. Flow field and noise characteristics of a supersonic impinging jet. Journal of Fluid Mechanics, 392:155-181, 1999.

11 Matteo Mancinelli, Vincent Jaunet, Peter Jordan, and Aaron Towne. Screech-tone prediction using upstream-travelling jet modes. Experiments in Fluids, 60(1):22, 2019.

10 of 11 
$12 \mathrm{G}$ Neuwerth. Acoustic feedback of a subsonic and supersonic free jet which impinges on an obstacle. NASA TT F-15719, 1974.

13 A. Powell. On the mechanism of choked jet noise. Proceedings of the Physical Society. Section B, 66(12): 1039, 1953.

14 Alan Powell. On edge tones and associated phenomena. Acta Acustica United with Acustica, 3(4):233-243, 1953.

15 Alan Powell. The sound-producing oscillations of round underexpanded jets impinging on normal plates. The Journal of the Acoustical Society of America, 83(2):515-533, 1988.

16 Ganesh Raman. Advances in understanding supersonic jet screech: Review and perspective. Progress in aerospace sciences, 34(1-2):45-106, 1998.

17 Oliver T Schmidt, Aaron Towne, Tim Colonius, André VG Cavalieri, Peter Jordan, and Guillaume A Brès. Wavepackets and trapped acoustic modes in a turbulent jet: coherent structure eduction and global stability. Journal of Fluid Mechanics, 825:1153-1181, 2017.

18 Hao Shen and Christopher K. Tam. Three-dimensional numerical simulation of the jet screech phenomenon. AIAA journal, 40(1):33-41, 2002.

19 C. K. W. Tam and F. Q. Hu. On the three families of instability waves of high-speed jets. Journal of Fluid Mechanics, 201:447-483, 1989.

20 Christopher KW Tam and KK Ahuja. Theoretical model of discrete tone generation by impinging jets. Journal of Fluid Mechanics, 214:67-87, 1990.

21 Aaron Towne, André VG Cavalieri, Peter Jordan, Tim Colonius, Oliver Schmidt, Vincent Jaunet, and Guillaume A Brès. Acoustic resonance in the potential core of subsonic jets. Journal of Fluid Mechanics, 825:1113-1152, 2017.

22 FR Wagner. The sound and flow field of an axially symmetric free jet upon impact on a wall. National Aeronautics and Space Administration, 1971.

23 Joel L Weightman, Omid Amili, Damon Honnery, Julio Soria, and Daniel Edgington-Mitchell. An explanation for the phase lag in supersonic jet impingement. Journal of Fluid Mechanics, 815, 2017.

24 Joel L Weightman, Omid Amili, Damon Honnery, Daniel Edgington-Mitchell, and Julio Soria. Nozzle external geometry as a boundary condition for the azimuthal mode selection in an impinging underexpanded jet. Journal of Fluid Mechanics, 862:421-448, 2019. 